\title{
BMJ Open Exploring the evidence for the effectiveness of health interventions for COVID-19 targeting migrants: a systematic review protocol
}

\author{
Stefania Mondello (D) , ${ }^{1}$ Carmela Visalli, ${ }^{1}$ Firas Kobeissy, ${ }^{1,2}$ Laura Cacciani (D),${ }^{3}$ \\ Fabio Cruciani, ${ }^{3}$ Stefania D'Amato, ${ }^{4}$ Anteo Di Napoli, ${ }^{5}$ Paolo Giorgi Rossi (1) ,' \\ Caterina Milli, ${ }^{7}$ Alessio Petrelli, ${ }^{5}$ Caterina Silvestri, ${ }^{7}$ Achille Cernigliaro, ${ }^{8}$ \\ Salvatore Scondotto ${ }^{8}$
}

To cite: Mondello S,

Visalli C, Kobeissy F, et al. Exploring the evidence for the effectiveness of health interventions for COVID-19 targeting migrants: a systematic review protocol. BMJ Open 2021;11:e057985. doi:10.1136/ bmjopen-2021-057985

- Prepublication history and additional supplemental material for this paper are available online. To view these files, please visit the journal online (http://dx.doi.org/10.1136/ bmjopen-2021-057985).

Received 06 October 2021 Accepted 25 November 2021

Check for updates

(C) Author(s) (or their employer(s)) 2021. Re-use permitted under CC BY-NC. No commercial re-use. See rights and permissions. Published by BMJ.

For numbered affiliations see end of article.

Correspondence to Prof Stefania Mondello; stm_mondello@hotmail.com

\section{ABSTRACT}

Introduction Owing to their inherent vulnerabilities, the burden of COVID-19 and particularly of its control measures on migrants has been magnified. A thorough assessment of the value of the interventions for COVID-19 tailored to migrants is essential for improving their health outcomes as well as promoting an effective control of the pandemic. In this study, based on evidence from primary biomedical research, we aimed to systematically identify health interventions for COVID-19 targeting migrants and to assess and compare their effectiveness. The review will be conducted within a programme aimed at defining and implementing interventions to control the COVID-19 pandemic in Italy, funded by the Italian Ministry of Health and conducted by a consortium of Italian regional health authorities.

Methods and analyses Data sources will include the bibliographic databases MEDLINE, Embase, LOVE Platform COVID-19 Evidence, and Cochrane Central Register of Controlled Trials. Eligible studies must evaluate health interventions for COVID-19 in migrants. Two independent reviewers will screen articles for inclusion using predefined eligibility criteria, extract data of retained articles and assess methodological quality by applying the Cochrane Risk of Bias tool. Disagreements will be resolved through consensus or arbitrated by a third reviewer if necessary. In synthesising the evidence, we will structure results by interventions, outcomes and quality. Where studies are sufficiently homogenous, trial data will be pooled and meta-analyses will be performed. Data will be reported according to methodological guidelines for systematic review provided by the Cochrane Collaboration and the Preferred Reporting Items for Systematic Reviews and Meta-Analyses statement.

Ethics and dissemination This is a review of existing literature, and ethics approval is not required. We will submit results for peer-review publication and present at relevant conferences. The review findings will be included in future efforts to develop evidence-informed recommendations, policies or programmatic actions at the national and regional levels and address future highquality research in public health.

\section{Strengths and limitations of this study}

- Our protocol, written according to the Preferred Reporting Items for Systematic Reviews and MetaAnalysis Protocols, ensures a rigorous and transparent approach capable of providing a trustworthiness and exhaustive evidence synthesis of the effectiveness of different health interventions for COVID-19 targeting migrants.

- Comprehensive and up-to-date coverage of the evidence will be ensured by the search and inclusion of data from preprints.

- Heterogeneity and a limited number of studies evaluating some interventions may prevent a quantitative synthesis of the evidence.

- Due to the rapidly changing epidemiological situation, including the spread of new variants and vaccination, results may not be applicable to the new scenarios.

\section{INTRODUCTION}

The COVID-19 pandemic has amplified health inequalities, particularly in populations with substantial vulnerabilities such as international migrants and refugees. ${ }^{12}$ Owing to their ethnic, social, economic and cultural diversity and precarious living conditions, these populations confront exclusion and multiple barriers to health services and care, experiencing a higher risk of infection and more devastating effects in the context of COVID-19. ${ }^{3}{ }^{4}$ Moreover, this situation complicates the control of the pandemic and an effective response, representing a considerable threat also to collective health and well-being. ${ }^{5}$

Many published articles have tackled the issue of developing the best possible management approach to leave no one behind, ${ }^{56}$ advocating for the needs of equitable and inclusive 
strategies, as well as health programmes focused on migrant and refugee populations. Accordingly, recommendation documents have been developed to guide migrant health interventions in relation to COVID-19 pandemic ${ }^{78}$; however, they are mainly based on evidence from studies on the general population that do not take into account the specific health needs and vulnerabilities, or the distinctive cultural and socioeconomic features, thereby possibly reducing the effectiveness.

There is now increased awareness and international interest in getting high-quality, trustworthy evidence on the efficacy and safety of health interventions for COVID-19 precisely targeting migrant populations to optimise health strategies, promote their acceptance and trust, and inform the development of tailored practice recommendations while guiding intervention allocation and prioritisation in both host and migrant populations.

The Italian Ministry of Health funded a project to be conducted by a consortium of regional health authorities and other scientific partners in order to exploit the potential of routinely collected data on the COVID-19 pandemic in Italy focusing on migrant health and to define and implement effective interventions to control the spread of infection and the burden of disease. The systematic review will be part of this project. In this study, we seek to systematically identify all studies evaluating health interventions for COVID-19 in migrant populations and methodically synthesise their efficacy across different settings and multiple outcomes. Migrants now represent a considerable proportion of most high-income countries' populations. ${ }^{9}$ This systematic review will provide a first clear foundation on which to base further primary research and produce evidence-based knowledge for future policy and clinical practice and for enhancing public health responses, thus helping to reduce health inequalities, implementing effective personalised interventions and, ultimately, maximising our chances for success in improving health status in migrants and in tackling the COVID-19 pandemic.

\section{METHODS}

The research question has been developed by a team of experts, including clinicians, researchers, methodologists and biostatisticians. The protocol has been designed in accordance with the methodological guidelines provided by the Cochrane Collaboration and the Preferred Reporting Items for Systematic Reviews and Meta-Analysis Protocols ${ }^{10}$ (the checklist is provided in online supplemental file 1), and it is registered in the International Prospective Register of Systematic Reviews (PROSPERO) (no. CRD42021282496, https://www.crd.york.ac.uk/ prospero/display_record.php?RecordID=282496). Any relevant amendments to this protocol will be updated in PROSPERO. We started developing the protocol on 1 August 2021, and the systematic review is expected to be completed in January 2022.

\section{Eligibility criteria}

\section{Types of studies}

For the purposes of this review, studies will be eligible if they evaluate and present the results of health interventions for COVID-19 targeting migrants. Any study without a comparative evaluation (defined as including a parallel comparison or control groups or using a before/after design) will be excluded. Study protocols will also be excluded. No restrictions will be applied based on publication status (peer-reviewed, in press or preprint), setting or language.

\section{PICO (population, intervention, comparison, and outcome) criteria}

\section{Types of participants}

The population of interest is migrants or international migrants, defined as foreign-born/foreign citizens in high-income countries, and including refugees, asylum seekers, economic migrants-labour migrants (both skilled and unskilled), and undocumented/illegal migrants living temporarily (at least a year) or permanently outside their country of origin.

\section{Types of interventions}

This systematic review will include studies evaluating any health intervention (ie, any strategy designed to produce behaviour changes or improve health status) for COVID-19 in migrants. The interventions can be delivered in any setting (healthcare, social or community setting), but they must specifically target migrants. Those aimed at the general population will be excluded.

\section{Types of comparisons}

We will include any comparisons, control groups, counterfactual or prestudies-poststudies.

\section{Type of outcomes measures}

Included studies are required to report outcome measures gauging the efficacy of the provision of health intervention for COVID-19 in migrants. These may include but are not limited to the following: SARS-CoV-2 infection, mortality, morbidity, mechanical ventilation, duration of hospital stays, intensive care unit length of stay, time to symptom resolution or clinical improvement, or the number of vaccine doses administered.

\section{Search strategy}

A search strategy template has been developed with assistance from an expert information specialist (see online supplemental file 2). The following electronic databases have been searched for potentially eligible studies: MEDLINE, Embase, LOVE Platform COVID-19 Evidence and Cochrane Central Register of Controlled Trials from 2019 to 5 November 2021, which will be updated to prior manuscript submission. In addition to these databases, reference lists of included articles, reference lists of relevant systematic reviews, clinicaltrials. gov, a PubMed-related article search, and OpenGrey will be searched. References will be managed using the 


\section{Inclusion criteria}

Clinical studies (experimental or observational) evaluating health interventions (ie, any strategy designed to produce behaviour changes or improve health status) for COVID-19 targeting migrants and refugees

No restrictions will be applied based on publication status, the severity of illness, setting or language.

\section{Exclusion criteria}

Lack of a comparative evaluation (ie, control groups, counterfactual, or predesign-postdesign)

Study protocols, case reports, narrative comments and animal studies citation management software EndNote V.X9 (Thomson Reuters).

\section{Process for study screening and selection}

After removing duplicates, titles and abstracts will be independently screened against the eligibility criteria (table 1) by two authors. Discrepancies between the two review authors regarding study eligibility will be resolved by discussion and consensus and, if necessary, by a third reviewer. In case of disagreement, a third reviewer will be consulted. The same process will be repeated on the full text to confirm inclusion in the systematic review. A table of excluded studies will be attached as an appendix.

\section{Data extraction}

Data will be extracted independently by two review authors and recorded using a standardised data collection form. Disagreements between reviewers will be discussed until consensus is reached, and, if necessary, a third reviewer will be consulted for arbitration.

The following information will be extracted: (1) bibliographic details (author, publication year and journal name); (2) details and methodological characteristics of the study (study design and aim, eligibility criteria, location and setting); (3) population characteristics (number of participants total, subjects demographics and migrant status); (4) intervention characteristics (type of intervention, doses, timing and duration, and mode of delivery); (5) outcome data (outcome type and timing of assessment); (6) results (mean/SD or number of events/ number of participants, effect estimate (eg, relative risk and odds ratio), 95\% CIs and SE); and (7) sources of funding and declarations of conflicts of interest. We will contact the authors of the included studies for clarification or to retrieve any missing data.

\section{Assessment of methodological quality of included studies}

The methodological quality of the studies retrieved through our search and included in the systematic reviews will be evaluated by two review authors independently using the Cochrane Risk of Bias tool. ${ }^{11}$ To assess the risk of bias in non-randomised trial designs, the New Castle Ottawa Scale will be used. ${ }^{12}$ Discrepancies in the evaluations will be resolved by consensus between the authors and, if necessary, arbitration by a third author. The risk of bias assessment will be integrated into the results and conclusions of the overview. More specifically, studies will not be excluded based on the quality assessment; however, if applicable, we will use the quality assessment to inform sensitivity analyses and to explore the consequences of synthesising studies of differing quality.

\section{Summary synthesis}

We will first summarise the characteristics of included studies in tables, including study design, participant and intervention characteristics. We will structure systematic review results by interventions and outcomes, and then by quality (ie, low to high risk of bias). We will tabulate results by the presentation of effect estimates and $95 \%$ CIs, and present the results of the risk of bias assessment using a 'risk of bias' graph figure and a risk of bias summary figure. Furthermore, we will identify methodological problems and gaps to inform and make recommendations for future clinical research.

We anticipate that some interventions will be evaluated by few studies, and there will be a range of different outcome measures reported across included studies, which may make meta-analysis of the data inappropriate, in which case the findings of included studies will be presented in a narrative synthesis. Where suitable data are available, we will perform a meta-analysis using RevMan V.5.4 software. Statistical heterogeneity will be assessed using the I-squared $\left(\mathrm{I}^{2}\right)$ statistic. ${ }^{13}$ Inconsistency across studies will be considered low, moderate and high for $\mathrm{I}^{2}$ values lower than $25 \%$, between $25 \%$ and $50 \%$, and greater than $50 \%$, respectively. Where substantial heterogeneity is detected $\left(\mathrm{I}^{2} \geq 50 \%\right)$, possible explanations will be investigated informally, and, if appropriate, the data will be analysed using a random-effects model. ${ }^{14}$ When sufficient data are reported, we will conduct subgroup analyses based on the following categories: (1) demographics (age, gender, ethnicity and socioeconomic status); (2) clinical characteristics (multimorbidity); (3) epidemiological phase context (low/high virus circulation; low/high vaccine coverage; dominant virus variant, ie, wild type, alpha, delta and others); (4) intervention characteristics (determined based on the interventions included); and (5) geographical areas (eg, Asia, Africa, Europe, South and North America). If appropriate, we will conduct sensitivity analyses by excluding studies of high risk of bias. We will also examine the effects of any outliers by omitting them from the analysis. Based on the quality of the evidence, we will produce a hierarchical list of interventions. 


\section{Assessment of the certainty of the evidence}

We will use the Grading Recommendation Assessment, Development and Evaluation (GRADE) ${ }^{15}$ to assess the certainty of the evidence for each outcome as emerging from the analysis of the included studies. The certainty of the evidence will be downgraded by one (serious concern) or two (very serious concerns) for the following reasons: risk of bias, inconsistency (unexplained heterogeneity and inconsistency of results), indirectness (indirect population, intervention, control and outcomes), imprecision (wide CIs) and publication bias. Judgements about the certainty of the evidence (high, moderate, low or very low) will be justified and incorporated into the results for each outcome. We will use GRADEpro GDT: GRADEpro Guideline Development Tool (Software) (McMaster University, 2020, developed by Evidence Prime, available from gradepro.org) to create 'Summary of findings' tables. The certainty of the evidence assessments will be integrated into the results and conclusions of the systematic review.

\section{PATIENT AND PUBLIC INVOLVEMENT}

This systematic review does not include any patient and public involvement.

\section{ETHICS AND DISSEMINATION}

No ethics approval is necessary when conducting a systematic review. The findings will be part of the project reporting to the Italian Ministry of Health and the regional health authorities of the participating regions. The findings will be also published in a peer-reviewed medical journal and presented at relevant national and international conferences. Other forms of dissemination will include direct engagement with policymakers, healthcare professionals, researchers and migrant representatives. We will also disseminate the results through specific forums, Twitter and other social media outlets to maximise impact.

\section{DISCUSSION}

There is an urgent need to ensure effective COVID-19 services and interventions for migrants, adapted to their individual (demographic, clinical and living conditions) characteristics and needs. ${ }^{2}{ }^{21-18}$ As a result, it is necessary to produce comprehensive and rigorous summaries to guide future policy, practice and research. Nonetheless, to the best of our knowledge, there is no systematic review synthetising the existing literature on COVID-19 interventions aimed at improving health outcomes among migrants. Our work will provide solid trustworthiness evidence synthesis, systematically examining the characteristics of the studies, health interventions and migrant populations in the published literature, as well as gauging the effectiveness and quality and describing the methods used and limitations. The commitment by the Ministry of Health and the participation of the regional health authorities in the project will ensure a certain bridging from the evidence synthesis to the decision-making process, at least in the Italian context.

Considering the growing size of migrant populations and the more recent immigration surge/refugee crisis after Taliban takeover, the conclusions drawn from the present review can make a difference in these vulnerable populations by helping policymakers, healthcare providers and researchers identify key actions and effective intervention approaches while also ensuring equitable access to healthcare services.

\section{Author affiliations}

${ }^{1}$ Department of Biomedical and Dental Sciences and Morphofunctional Imaging, University of Messina, Messina, Italy

${ }^{2}$ Department of Biochemistry and Molecular Genetics, American University of Beirut Medical Center, Beirut, Lebanon

${ }^{3}$ Dipartimento di Epidemiologia, SSR Lazio, Azienda Sanitaria Locale Roma 1, Roma, Italy

${ }^{4}$ Ministry of Health, Rome, Italy

${ }^{5}$ INMP, National Institute for Health, Rome, Italy

${ }^{6}$ Servizio Interaziendale Epidemiologia, AUSL Reggio Emilia, Reggio Emilia, Italy

${ }^{7}$ Agenzia Regionale di Sanità della Toscana, Firenze, Toscana, Italy

${ }^{8}$ Department of Health Services and Epidemiological Observatory, Regional Health

Authority, Palermo, Italy

Contributors SM, CV, FK, LC, FC, SDA, ADN, PGR, CM, AP, CS, AC and SS developed the systematic review protocol; SM drafted the manuscript; CV, FK, LC, FC, SDA, $A D N, P G R, C M, A P, C S, A C$ and SS critically revised the manuscript; SM, CV, FK, LC, $\mathrm{FC}, \mathrm{SDA}, \mathrm{ADN}, \mathrm{PGR}, \mathrm{CM}, \mathrm{AP}, \mathrm{CS}, \mathrm{AC}$ and $\mathrm{SS}$ have approved the final version.

Funding This work was supported by the Italian Ministry of Health (CCM Program 2020, award/grant number is not applicable).

Competing interests None declared.

Patient consent for publication Not applicable.

Provenance and peer review Not commissioned; externally peer reviewed.

Supplemental material This content has been supplied by the author(s). It has not been vetted by BMJ Publishing Group Limited (BMJ) and may not have been peer-reviewed. Any opinions or recommendations discussed are solely those of the author(s) and are not endorsed by BMJ. BMJ disclaims all liability and responsibility arising from any reliance placed on the content. Where the content includes any translated material, BMJ does not warrant the accuracy and reliability of the translations (including but not limited to local regulations, clinical guidelines, terminology, drug names and drug dosages), and is not responsible for any error and/or omissions arising from translation and adaptation or otherwise.

Open access This is an open access article distributed in accordance with the Creative Commons Attribution Non Commercial (CC BY-NC 4.0) license, which permits others to distribute, remix, adapt, build upon this work non-commercially, and license their derivative works on different terms, provided the original work is properly cited, appropriate credit is given, any changes made indicated, and the use is non-commercial. See: http://creativecommons.org/licenses/by-nc/4.0/.

ORCID iDs

Stefania Mondello http://orcid.org/0000-0002-8587-3614

Laura Cacciani http://orcid.org/0000-0002-5800-3285

Paolo Giorgi Rossi http://orcid.org/0000-0001-9703-2460

\section{REFERENCES}

1 Lau LS, Samari G, Moresky RT, et al. COVID-19 in humanitarian settings and lessons learned from past epidemics. Nat Med 2020;26:647-8.

2 Orcutt M, Patel P, Burns R, et al. Global call to action for inclusion of migrants and refugees in the COVID-19 response. Lancet 2020;395:1482-3. 
3 Sze S, Pan D, Nevill CR, et al. Ethnicity and clinical outcomes in COVID-19: a systematic review and meta-analysis. EClinicalMedicine 2020;29:100630.

4 Hayward SE, Deal A, Cheng C, et al. Clinical outcomes and risk factors for COVID-19 among migrant populations in high-income countries: a systematic review. J Migr Health 2021;3:100041.

5 Zard M, Lau LS, Bowser DM, et al. Leave no one behind: ensuring access to COVID-19 vaccines for refugee and displaced populations. Nat Med 2021;27:747-9.

6 Orcutt M, Spiegel P, Kumar B, et al. Lancet Migration: global collaboration to advance migration health. Lancet 2020;395:317-9.

7 WHO. Interim guidance for refugee and migrant health in relation to COVID-19 in the WHO European Region, 2020. Available: https:// www.euro.who.int/_data/assets/pdf_file/0008/434978/Interimguidance-refugee-and-migrant-health-COVID-19.pdf

8 The new humanitarian, 2021. Available: https://www. thenewhumanitarian.org/maps-and-graphics/2021/coronavirushumanitarian-updatemap-data-vaccine-aid-response

9 Abubakar I, Aldridge RW, Devakumar D, et al. The UCL-Lancet Commission on migration and health: the health of a world on the move. Lancet 2018;392:2606-54.

10 Shamseer L, Moher D, Clarke M, et al. Preferred reporting items for systematic review and meta-analysis protocols (PRISMA-P) 2015: elaboration and explanation. BMJ 2015;350:g7647.
11 Higgins JPT, Green S, eds. Cochrane Handbook for Systematic Reviews of Interventions. The Cochrane Collaboration, 2011.

12 Wells GA, O'Connell D, Peterson J, et al. Newcastle-Ottawa quality assessment scale. Ottawa Hosp Res Institute 2014;3:2-4.

13 Higgins JPT, Thompson SG. Quantifying heterogeneity in a metaanalysis. Stat Med 2002;21:1539-58.

14 Deeks JJ, Higgins JPT, Altman DG. Chapter 9: Analysing data and undertaking meta-analyses. In: Higgins JPT, Green S, eds. Cochrane Handbook for systematic reviews of interventions version 5.1.0. The Cochrane Collaboration, 2011.

15 Guyatt GH, Oxman AD, Vist GE, et al. Grade: an emerging consensus on rating quality of evidence and strength of recommendations. BMJ 2008;336:924-6.

16 Hargreaves S, Kumar BN, McKee M, et al. Europe's migrant containment policies threaten the response to covid-19. BMJ 2020;368:m1213.

17 Hargreaves S, Zenner D, Wickramage K, et al. Targeting COVID-19 interventions towards migrants in humanitarian settings. Lancet Infect Dis 2020;20:645-6.

18 Greenaway C, Hargreaves S, Barkati S, et al. COVID-19: exposing and addressing health disparities among ethnic minorities and migrants. J Travel Med 2020;27:taaa113. 